\title{
Metabolomics for metabolically manipulated plants: effects of tryptophan overproduction
}

\author{
Atsushi Ishihara $\cdot$ Fumio Matsuda $\cdot$ Hisashi Miyagawa \\ Kyo Wakasa
}

Received: 21 May 2007 / Accepted: 28 June 2007/Published online: 1 September 2007

(C) Springer Science+Business Media, LLC 2007

\begin{abstract}
Advances in molecular breeding technologies have enabled manipulation of the concentrations of specific plant components by modifying the genes that play a key role in their production. This has provided new opportunities to enhance the nutritional quality of major crops. However, given that metabolic pathways form a highly integrated network, any alteration in a given biosynthetic pathway is most likely to effect secondary and unpredicted changes in the metabolite profile of other pathways. Metabolomics technologies can contribute to the efficient detection of such unexpected effects caused by genetic modification. This has relevance not only from the perspective of safety evaluations of newly developed crops, but to basic science focused on uncovering hitherto unknown regulatory mechanisms associated with the biosynthesis and catabolism of primary and secondary metabolites in plants. In this review, recent advances in plant metabolic engineering for the overproduction of
\end{abstract}

A. Ishihara $\cdot$ F. Matsuda $\cdot$ H. Miyagawa $\cdot$ K. Wakasa

CREST, Japan Science and Technology Agency,

Tokyo 103-0027, Japan

\author{
A. Ishihara $\cdot$ F. Matsuda $\cdot$ H. Miyagawa \\ Division of Applied Life Sciences, Graduate School of \\ Agriculture, Kyoto University, Kyoto 606-8502, Japan \\ K. Wakasa $(\bowtie)$ \\ Department of Agriculture, Tokyo University of Agriculture, \\ 1737 Funako, Atsugi, Kanagawa 243-0034, Japan \\ e-mail: k3wakasa@nodai.ac.jp \\ Present Address: \\ F. Matsuda \\ Metabolome Research Group, RIKEN Plant Science Center, \\ Yokohama, Kanagawa 230-0045, Japan
}

tryptophan (Trp), one of the essential amino acids, are described. In particular, the efficacy of a transgene OASAID that encodes a mutant anthranilate synthase (AS) $\alpha$ subunit of rice in specifically elevating levels of Trp without marked secondary effects on the metabolite profile of rice is demonstrated. Related topics, such as regulation of Trp biosynthesis, possible interactions between the biosyntheses of Trp and other aromatic amino acids, and translocation of Trp in are discussed based on findings derived from metabolomic analyses of Trp-overproducing transgenic plants.

Keywords Anthranilate synthase - Metabolomics · OSA1AD · Rice · Tryptophan

\section{Introduction}

Historically, plants have represented a critical nutritional and valuable life resource not only for human beings but for the domesticated animals they feed. Hence, enormous efforts are expended on improving the qualities and character of major crops. Whilst this improvement has relied on classical breeding techniques for most of the earlier historical period, recent advances in recombinant gene technology have made it possible to manipulate or improve the properties of plants in a relatively short time span. Genetic modification of crops through recombinant technology can be categorized by the following three goals.

The first goal centers on endowing crops with properties typically absent in plants. For example, (1) crops producing insecticidal Bt toxins of bacterial origin have been developed to enhance herbivore resistance, and are now cultivated worldwide (Shelton et al. 2000); (2) a gene encoding 
an enzyme (5-enolpyruvylshikimate-3-phosphate synthase) that is insensitive to a specific herbicide is utilized to generate herbicide-resistant crops, thus alleviating weeding labor in the field (Dill 2005); (3) production of a biodegradable plastic polymer, poly- $\beta$-hydroxybutyrate (PBH), is undertaken by transforming plants with a series of bacterial genes involved in PBH production (Saruul et al. 2002).

In a second goal, genetic modification is applied to improve the productivity of beneficial components that are present only in a trace amount. Targets include, not only essential amino acids and vitamins that are associated with the nutritional value of crops, but also secondary metabolites of pharmaceutical value (see sections below for further discussion on how understanding the regulation of biosynthesis of a given plant component is critical to efficacious enhancement of its production).

Third, one can attempt to decrease the amount of harmful or wasteful components in plants. For example; (1) high levels of certain glucosinolates in Brassica can restrict their use as food and feed, and hence, reduction of their amounts by altering metabolic flux through their biosynthetic pathway is a goal (Chavadej et al. 1994); (2) modification of the content of lignin has attracted considerable attention, as lignin disturbs both the paper manufacturing process and digestion of feed crops in farm animals (Boudet andGrima-Pettenati 1996).

For all three major goals, manipulation of metabolic function in a plant can cause changes in the activity, not only of the target pathway, but also of multiple pathways interacting directly or indirectly with the target pathway. Such interactions may involve, for example, competition for common precursors. When the activity of a target pathway is enhanced, this could result in the decrease in substrate supply to a competing pathway. On the other hand, suppression of a target pathway may result in increased substrate supply for a competing pathway. Any impact on feedback regulation through altered substrate levels is also likely to have pronounced and potentially more complicated effects on metabolite composition.

In this review, we survey recent advances in plant metabolic engineering as a means to enhance the nutritional value of major crops. We also specifically discuss our recent successes in increasing the production of the essential amino acid, tryptophan (Trp), utilizing a mutant gene encoding the anthranilate synthase (AS) $\alpha$ subunit. The effects of activating Trp biosynthesis on the composition of the plant metabolome will be discussed in the context of our recent metabolite profiling analyses and to provide more general lessons for the future generation of genetically modified crops.

\section{Increasing the nutritional value of crop plants by metabolic engineering}

Most animals, including humans, rely primarily on plants as a source of carbon. Plants, of course, can fix inorganic carbon to edible organic forms through photosynthesis. Enhancement of photosynthetic activity to produce and accumulate increased levels of carbohydrate has therefore represented one of the most important research subjects in metabolic engineering of crops (Matsuoka et al. 2001; Nunes-Nesi et al. 2005; Parry et al. 2007). Increasing seed oil content is also an issue of primary concern though, generally, breeding efforts are more typically directed to modifying fatty acid composition as a means to improve oil quality (Thelen and Ohlrogge 2002).

Plants are also a valuable nitrogen source. For example, animals cannot synthesize many of the amino acids required for protein synthesis. Whilst carnivorous animals acquire amino acids and other organic nitrogen compounds by devouring their prey, herbivorous and omnivorous animals are dependent on plants for their supply of these essential amino acids. Yet plants do not always contain sufficient amounts of these essential amino acids and this provides the basis for research programs focused on increasing their levels through metabolic engineering. Representative essential amino acids include lysine (Lys), methionine (Met), threonine (Thr), and Trp. The endogenous levels of these amino acids are regulated by a complex system of feedback controls operating at key steps within each pathway. Premised on an understanding of such mechanisms, deregulation of feedback control represents a promising approach to increase levels of Lys, Met, and Thr. This deregulation typically involves incorporation of a microbially derived feedback insensitive (or less-sensitive) version of a critical biosynthetic enzyme. Overexpression of genes encoding seed proteins that are rich in essential amino acids also represents a popular strategy to raise amino acid levels (reviewed by Dewaele et al. 2002; Galili and Höfgen 2002; Galili et al. 2005).

Plants are also a primary source of trace nutrients, or vitamins, and these metabolites have been an important target group for metabolic engineering. Efforts to enhance the production of folate and vitamins $\mathrm{A}, \mathrm{C}$, and $\mathrm{E}$ by the insertion of one or two genes encoding the key enzyme(s) in each biosynthetic pathway have been reported (Hossain et al. 2004, and the references therein). Transgenic rice with enhanced provitamin A ( $\beta$-carotene) biosynthetic properties is referred to as "Golden Rice" and is predicted to markedly contribute to alleviating vitamin A deficiency among people relying on rice as a primary food staple (Beyer et al. 2002; Stein et al. 2006). 
3 Potential for secondary changes caused by metabolic engineering

Enhancement of crop quality by altering the concentration of a target metabolite is usually achieved by modification of the relevant biosynthetic pathway. However, plants have evolved effective metabolic regulation to avoid wasteful utilization of fixed carbon and the production of unnecessary metabolites. Thus, deregulation of key biosynthetic steps is required for metabolic engineering to be successful. However, such deregulation can be anticipated to impact many aspects of plant metabolism and, in most cases, such secondary effects will be unintended and impossible to predict. Possible factors that can cause these secondary changes are now discussed.

\subsection{Unexpected function of the gene product}

A newly introduced enzyme may catalyze a reaction that does not normally occur in the original species. Substrate specificity will define the number and range of compounds an enzyme can accept. Should a newly introduced enzyme encounter an acceptable substrate that is not present in the original species it can catalyze unintended reactions. Incomplete knowledge of the substrate specificity of any introduced enzyme can render it difficult to predict the scope of secondary metabolic changes in transgenic plants. Murooka et al. (2002) introduced the soybean aspartate (Asp) aminotransferase gene, AAT5, into Arabidopsis. The encoded enzyme catalyzes the transfer of an amino group from glutamate (Glu) to oxalacetate to form Asp and 2oxoglutarate. However, expression of AAT5 in Arabidopsis also caused increases in levels of alanine (Ala) and glycine (Gly). These two amino acids could be formed if the AAT5 enzyme also catalyzed the transfer of an amino group from Glu to pyruvate and glyoxylate, respectively, in a manner analogous to the original reaction. On the basis of these hypothetical reaction schemes, Murooka et al. (2002) speculated that AAT5 has broad substrate specificity allowing catalytic formation of Ala and Gly when expressed in Arabidopsis.

\subsection{Metabolism of newly elevated compounds}

A host plant may possess innate ability to convert the product of an enzyme encoded by any introduced gene. For example, the expression of bacterial 4-hydroxycinnamoylCoA hydratase/lyase in tobacco was shown to result in increased production of glucosides of benzoic acid derivatives (Mayer et al. 2001). The most likely explanation for this is as follows. The expected product of the 4-hydroxycinnamoyl-CoA hydratase/lyase reaction is benzaldehyde. This is then oxidized, enzymatically or non-enzymatically, in tobacco to yield benzoate followed by conversion to the glucoside conjugates by endogenous glucosyltransferase.

\subsection{Effects on branching pathways}

Plant secondary metabolic pathways generally form a cascade by repeated branching. Depending on the position of a target step these complex branching cascades can give rise to unpredictable secondary consequences when subjected to metabolic engineering. This is now illustrated below.

\subsubsection{Modification of an upstream step of the branching point}

The modification of an upstream step of a branching point for two distinct pathways may affect metabolic flux in both pathways. The plant hormone indole-3-acetic acid (IAA) is biosynthesized from Trp via indole-3-acetaldoxime (IAOx). In Arabidopsis, formation of IAOx is catalyzed by the cytochrome P450 enzymes, CYP79B2 and CYP79B3 (Hull et al.2000; Mikkelsen et al. 2000). In addition, Arabidopsis contains the Trp derived secondary metabolites, indole glucosinolates (IGs), which are also biosynthesized via IAOx. IGs are mainly present in members of the Brassicaceae family, and have been implicated in defense against herbivorous insects and pathogens by generating toxic degradation products such as isothiocyanates after hydrolysis by thioglucosidase (myrosinase). The overexpression of CYP79B2 was shown to result in increased levels of both free IAA (Zhao et al. 2002) and IGs (Mikkelsen et al. 2000). In contrast, however, the double mutant cyp79B2 cyp $79 B 3$ showed a phenotype typical of IAA deficiency and had decreased levels of both IAA and IGs (Zhao et al. 2002).

It is also possible, however, that modification of an enzymatic step upstream of a branching point may affect only one of the two branches. In plants, phenylalanine ammonia-lyase (PAL) plays an important role in supplying phenylalanine (Phe) to pathways associated with secondary metabolism. In Arabidopsis, this enzyme consists of multiple isoforms. A double mutant of pall and pal2 lacked three major flavonoids present in the wild-type, yet amounts of sinapoyl glucose and sinapoyl matate were not altered (Rohde et al. 2004). The mechanism for the differential effect on these two downstream branches has yet to be elucidated.

\subsubsection{Modification of a downstream step of the branching point}

Changes in the levels of a single metabolite derived from one branching pathway can be effected by enzymatic changes in a competing branch. In other words, 
enhancement of one pathway can cause competition between the enhanced pathway and an associated branch pathway. In tobacco plants expressing bacterial hydroxycinnamoyl-CoA hydratase/lyase, the accumulation of glucosides of benzoic acid derivatives, as described above, was accompanied by a marked decrease in the contents of phenylpropanoids and flavonoids. This is because the newly introduced enzyme consumes hydroxycinnamoylCoA, the precursor for phenylpropanoid and flavonoid synthesis (Mayer et al. 2001).

This type of competition can be purposely exploited for reducing levels of undesired metabolites. Chavadej et al. (1994) introduced the Trp decarboxylase (TDC) gene from Catharanthus roseus into rapeseed in order to reduce the contents of IGs which interfere with the quality of protein meal The intent was to reduce metabolic flow to IGs through the creation of a new branch of Trp-derived secondary metabolism. Indeed, the IG content in mature seeds in the transgenic rapeseed expressing TDC was only 3\% of that found in untransformed seeds (see section below for further discussion). Analogously, the introduction of the TDC gene into potato resulted in reduction in the levels of Phe and chlorogenic acid (Yao et al. 1995). Phe and chlorogenic acid originate the shikimate pathway in common with Trp.

In contrast to the above examples, suppression of a given pathway can result in enhancement of an associated branched pathway. In the case of IAA and IG biosynthesis, loss-of-function mutations in the IG specific steps were shown to cause channeling of metabolic flow into IAA biosynthesis in Arabidopsis. In the IG pathway, IAOx is converted into indol-3-ylmethyl thiohydroxamate by the consecutive reactions catalyzed by CYP83B1 (Bak et al. 2001) and $C$-S lyase (Mikkelsen et al. 2004). sur2 and surl mutants with loss-of-function mutations in these genes were characterized by a "high-auxin" phenotype, and the sur2 mutant did indeed contain high levels of IAA (Barlier et al., 2000; Delarue et al. 1998). Conversely, overexpression of cyp83B1 led to auxin deficiency in Arabidopsis (Bak et al. 2001).

\section{4 "Accidental" inhibition of other pathways by an elevated metabolite}

A more "accidental" effect of the modification of a pathway on another pathway has been suspected in some cases. As described above, overexpression of a TDC gene in rapeseed plant resulted in the reduction of IG levels, and this reduction was considered to be the result of competition between the tryptamine branch and the IG pathway (Chavadej et al. 1994). However, tryptamine, the product of the TDC reaction, was subsequently shown to bind to CYP83B2 protein and inhibit its enzymatic activity (Bak et al. 2001). This finding therefore allows another interpretation for the reduction of IGs in the transgenic plant whereby elevated levels of tryptamine may have inhibited the CYP83B2 ortholog in rapeseed.

Another indication of accidental inhibition of a metabolic pathway was obtained from the Arabidopsis ref2 mutant that is defective in cyp83Al gene (Hemm et al. 2003). This mutant shows reduced fluorescence in the leaves under UV light and contains reduced level of sinapoyl malate and altered lignin composition with lower percentages of syringyl units relative to wildtype. Positional cloning of the mutated gene revealed that REF2 encodes CYP83A1 which catalyzes the metabolism of Metderived aldoximes in the biosynthesis of aliphatic glucosinolates. The impaired function of CYP83A1 in the ref2 mutant most likely led to the accumulation of Met-derived aldoximes. Since some synthetic aldoximes were inhibitory to caffeic acid $O$-methyltranferase, it is the accumulation of Met-derived aldoximes that may be responsible for the altered phenylpropanoid phenotype.

\subsection{Effects expressed via multiple event sequences}

Modification of metabolic pathways can affect other pathways via complex sequences of multiple events including alterations in gene expression and protein synthesis. Whilst those events may comprise a plant regulatory system designed to counteract certain metabolic modifications it is frequently difficult to logically associate cause and effect.

The involvement of a complex event sequence was suggested in the suppression of flavonoid accumulation in the Arabidopsis pall pal2 double mutant (Rohde et al. 2004). Depletion of the three major flavonoids could not be explained by a shortage of substrate supply as feeding of cinnamic acid or $p$-coumaric acid did not restore flavonoid accumulation. Thus, the cause of flavonoid depletion in the double mutant is thought to be a negative effect on the enzyme activities associated with flavonoid synthesis rather than reduced substrate availability. Indeed, the genes encoding enzymes in the flavonoid pathway, including chalcone synthase and dihydroflavonol 4-reductase, were downregulated in pall mutant. Interestingly, it is also known that the pall pal2 double mutation affected the expression of various genes involved in amino acid and sugar metabolism and it is reasonable to suggest that these changes may be signs of molecular adjustments to the metabolic changes caused by the mutation.

\section{Methodology of metabolite profiling of plants}

As detailed above, manipulation of the endogenous levels of a plant component by modifying regulation of a key 
enzyme(s) responsible for its biosynthesis will have some secondary effect, expected and unexpected, on the metabolism of other components. From a practical point of view, monitoring of unexpected effects of metabolic engineering is clearly of great importance and metabolic profiling analyses that cover the widest range of metabolites may prove particularly informative. For this purpose, non-targeted methods based on GC-MS (Fiehn et al. 2000; Garrat et al. 2005; Roessner-Tunali et al. 2003), HPLC-UV (photodiode array detection) (Fraser et al.2000), HPLCMS (Huhman and Sumner 2002; Roepenack-Lahaye et al. 2004), ${ }^{1}$ H-NMR (Baker et al. 2006; Choi et al. 2004; Gall et al. 2003; Manetti et al. 2004; see also the review by Ward et al. 2007), and high-resolution MS (Hirai et al. 2004; Oikawa et al. 2006) have been applied. Given the wide chemical and quantitative diversity of plant metabolites, however, no single analytical methodology can provide a definitive and absolute metabolite profile. To address this, at least in part, some researchers have adopted a combination of the above techniques. For example, Defernez et al. (2004) utilized both NMR and HPLC-UV to investigate metabolism in genetically modified potatoes. Methods based on NMR and GC-MS appear to have been adopted as versatile and reliable approaches to profiling plant constituents. As a general rule, metabolic profiling analysis using GC-MS and NMR tend to covers primary metabolites such as sugars, amino acids, and organic acids, mainly because of their relatively high abundance in plants. Electrospray ionization (ESI) combined with LC-MS analysis is capable of covering considerably wide range of compounds, and is particular useful for the profiling of secondary metabolites (T. Yamada et al., 2007, submitted data). However, quantitative profiling by ESI-MS is generally hampered by ion suppression and approaches to minimizing this problem is needed (Bamba and Fukusaki 2006)

Despite the many limitations when compared to other sophisticated but rather costly techniques, HPLC-UV(PDA) still has merit in monitoring the change in the amounts of a wide range of secondary metabolites with distinct chromophores, such as alkaloids produced from Trp and phenylpropanoids. Indeed, depending on the chromophore, UV detection can be more sensitive and quantitative than MS methods. Metabolic profiling using LC-PDA can also be applied to the identification of unknown metabolites. Morino et al. (2005), for example, demonstrated that the amounts of several UV active metabolites were slightly increased in the rice calli expressing $O A S A 1 D$ transgene by the metabolic profiling using LC-PDA methods. Following the isolation procedure using $\mathrm{LC}$, the structures of metabolites were determined to be indole alkaloids based on the combination of spectroscopic analyses.
Statistical processing of the large datasets generated in metabolic profiling experiments and data mining also play important roles in metabolomics, and a number of commercial and freely distributed software for the data processing and mining are now available. Among the various multi-variate analysis techniques, principal component analysis (PCA) appears to be used most frequently in order to evaluate metabolic changes in GM crops (Choi et al. 2004; Fiehn et al. 2000; Gall et al. 2003). Evaluation of loading plots can be used to find those metabolites most affected by genetic modification and which most contribute to the genotype difference (Baker et al. 2006; Defernez et al. 2004; Taylor et al. 2002). Differential analysis has also been applied, in which the levels of metabolites between the untransformed and transformed plants are, respectively, compared (Garrat et al. 2005; Ishihara et al. 2006; Morino et al., 2005). The approach is able to detect the changes in the levels of minor metabolites by using simple statistical tests such as Student's $t$-test and ANOVA.

As discussed above, metabolic engineering can affect the regulation of other metabolic pathway beyond that of the target pathway. In principle, any increase or decrease in the levels of non-target metabolites levels should be closely proportional to the amount of change in the target metabolite. Thus, the correlation coefficient between the levels of the target metabolite and of other metabolites can be used for detecting metabolites whose levels are affected by genetic modification. Recently, it has been suggested that the levels of many metabolites in plants fluctuate in concert with each other with high-correlation coefficients and that the level of each metabolite is coordinately controlled within a correlation network (Morgenthal et al. 2006). It has also been demonstrated that the modification of plant metabolic pathway can affects the structure and membership of a correlation network by addition and/or removal of correlations (Weckwerth et al. 2004). Such changes may provide important clue and new insights into the regulation of plant metabolic pathways.

Of course, once changes in a metabolite profile have been revealed by appropriate data mining, any components that are responsible for the change have to be chemically identified. Profiling using MS or NMR can sometimes work effectively for identifying such components. However, the availability of standard compounds and well-annotated chemical libraries are critical for rapid results. The classical and laborious techniques of traditional natural product chemistry may often be required, such as isolation of a compound in a substantial amount, followed by multispectroscopic analysis (Morino et al. 2005), and chemical synthesis (T. Yamada et al., 2007, submitted data). Further accumulation of knowledge on the mass and NMR 
spectrometry of the various plant constituents is required for more detailed profiling of plant metabolomes.

\section{Engineering of Trp productivity in plants}

Trp represents not only an essential amino acid but also the precursor of various secondary metabolites of pharmaceutical value. Its levels in plants are, however, typically limiting. While the industrial production of most amino acids can be achieved by microbial fermentation from sugar and ammonia, Trp is one of those whose production efficiency is relatively low (Ikeda 2006). Elevating Trp levels in plants has therefore represented a major component in breeding research. To achieve this by molecular metabolic engineering technology requires an understanding of the biosynthesis of Trp in plants as well as regulatory mechanisms controlling its production. It has been demonstrated that feedback control of the activity of AS, a key enzyme in the Trp biosynthetic pathway plays a critical role in determining Trp levels in plants. This enzyme has therefore been considered as the most important target of manipulation for Trp overproduction, as will be described in more detail below. The role of metabolic profiling techniques in assessing secondary effects on metabolite composition caused by Trp overproduction will also be discussed.

\subsection{Trp biosynthesis, regulation, and Trp related secondary metabolism}

The Trp is one of the aromatic amino acids biosynthesized by the so-called shikimate pathway, which starts from the condensation of erythrose-4-phosphate with phosphoenol pyruvate to give Phe and Tyr besides Trp. The pathway branches after the formation of chorismate from shikimate into two routes: One leads to Phe and Tyr, while the other provides Trp via an important intermediate, anthranilate (Fig. 1). Anthranilate is formed from chorismate by the catalytic action of AS. Anthranilate is then converted into Trp by five step reactions. For the synthesis of one molecule of Trp from chorismate, a single molecule of Gln and serine (Ser) is consumed. The final product Trp inhibits AS to regulate its production (Belser et al. 1971; Widholm 1972).

The Trp serves not only as a component of proteins, but also as a precursor of various secondary metabolites in plants (Fig. 2). IAA, a representative of the plant hormone auxin, is the most ubiquitous metabolite derived from Trp, although the presence of a Trp-independent biosynthetic pathway has been proposed. Other well known secondary metabolites of Trp origin include the pharmacologically active indole alkaloids such as vinblastine and camptothecin, and IGs (Hull et al. 2000; Mikkelsen et al. 2000; Zhao et al. 2002) that are typically found in Brassicaceae. The latter compounds are considered to play a defensive role in the producer plants against herbivores and phytopathogens. There are also many plant defense substances derived from Trp or intermediates in the Trp biosynthetic pathway: Gramine in barley (O'Donovan and Leete 1963), benzoxazinones in wheat and corn (Frey et al. 1997; Nomura et al. 2002), camalexin in Arabidopsis (Glawischniget al. 2004), avenacins (A. Ishihara et al. unpublished data), and avenanthramides in oats (Ishihara et al. 1999; Matsukawa et al. 2002), acridone alkaloids in Ruta graveolens (Gröger and Johne 1968).

As described above, AS plays a key role in the biosynthesis of Trp and catalyzes the formation of anthranilate from chorismate and Gln. The enzyme is a heterotetramer that consists of two sets of $\alpha$ and $\beta$ subunits (Bohlmann et al. 1995; Niyogi and Fink 1992; Niyogi et al. 1993; Poulsen et al. 1993). The $\beta$ subunit functions to liberate an ammonia molecule from Gln, which is transferred to chorismate by the action of the $\alpha$ subunit to form anthranilate and pyruvate. Of these two subunits, the $\alpha$ subunit is deemed to play a crucial role in the overall reaction, because the $\alpha$ subunit alone can function to slow conversion of chorismate to anthranilate in the presence of inorganic ammonia. Additionally, the binding site of Trp for feedback inhibition is present in the $\alpha$ subunit. To date, genes encoding AS have been isolated from a large number of microorganisms including bacteria, yeasts and fungi, as listed in available databases (for example, see http://www.genome.jp/kegg/genes.html). The crystal structures of AS from Serratia marcescens, a mesophilic bacterium (Spraggon et al. 2001), and Salmonella typhimurium (Morollo and Eck 2001) have been solved in the presence of substrates or an allosteric inhibitor, allowing structural characterization of the catalytic site as well as the Trp binding site. No crystal structure has been solved for AS of plant origin, although the homology between AS of plants and microorganisms is generally high.

To date, AS genes have been cloned from tobacco (Song et al. 1998), R. graveolens (Bohlmann et al., 1995), Arabidopsis (Niyogi and Fink 1992), rice (Tozawaet al. 2001) and oat (A. Ishihara et al. unpublished data), and the presence of two types of genes has been demonstrated in each of these plants. In some cases, one of the multiple AS family members in a plant is insensitive to feedback inhibition (Bohlmann et al.1996). Some AS enzymes are also inducible by stresses such as infection of pathogens and wounding stresses, inferring their possible role in the defense reaction (Bohlmann et al. 1995; Niyogi and Fink 1992). 


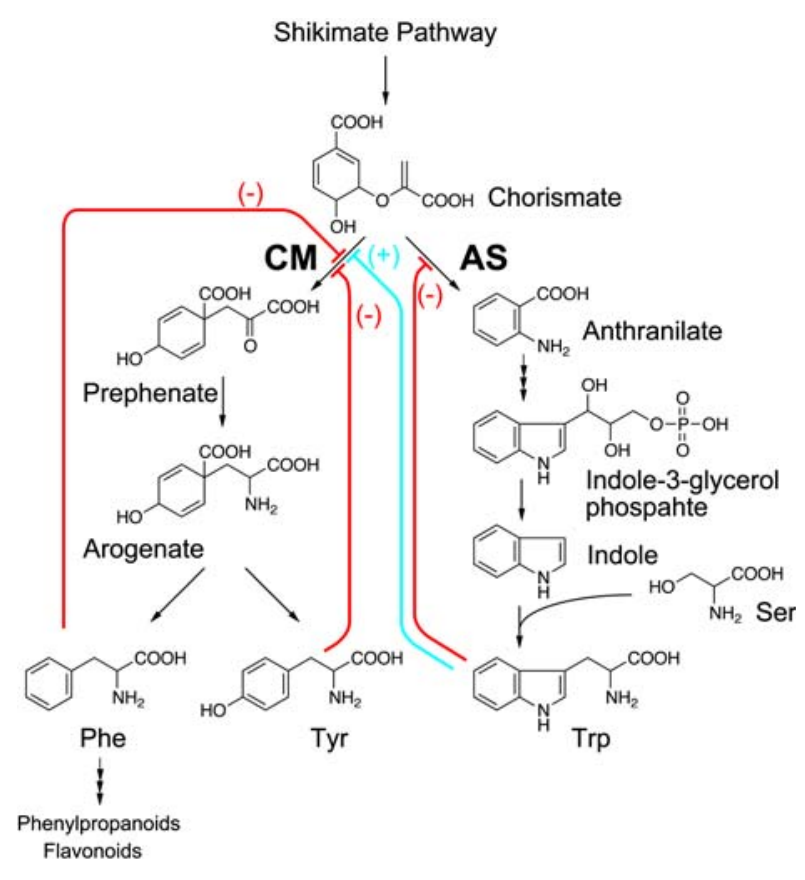

Fig. 1 Biosynthetic pathways for aromatic amino acids

\subsection{Enhancement of Trp production in plants}

Based on the role of feedback regulation in controlling endogenous amino acid concentrations, utilization of the feedback-insensitive version of AS has been a prominent feature of metabolic engineering attempts to increase Trp levels. For example, the feedback-insensitive ASA2 of tobacco was introduced and expressed in Astragalus sinicus (Legum) and in cultured hairy root of soybean as well as in tobacco itself. This resulted in 3-26-fold increases in the levels of Trp (Cho et al. 2000; Cho et al. 2004; Tsai et al. 2005; Zhang et al. 2001).

Screening for mutants that are resistant to Trp analogs can also identify AS feedback mutants. One such mutant is Arabidopsis trp-5, in which the gene ASA1, encoding one of the two AS $\alpha$ subunit isoforms, has a point mutation. This single base substitution causes a change in the amino acid sequence converting Asp-341 into Asn-341. This single change markedly reduces the sensitivity of AS activity to elevated levels of tissue Trp (Kreps et al. 1996; Li and Last 1996) and, consequently, levels of free Trp are increased threefold. In maize, a mutated AS $\alpha$ gene was isolated from a Trp-analog resistant line. When expressed in calli a tenfold increase in Trp levels was observed. Elevated levels of Trp (3.7-fold) were also observed in the kernels obtained from progenies of the plants regenerated from the transgenic calli (Anderson et al. 1997).

Inspired by these findings, attempts to generate plants with high-Trp content by gene manipulation were undertaken. For example, the mutant ASAI gene of Arabidopsis
Fig. 2 Trp-pathway derived secondary metabolites

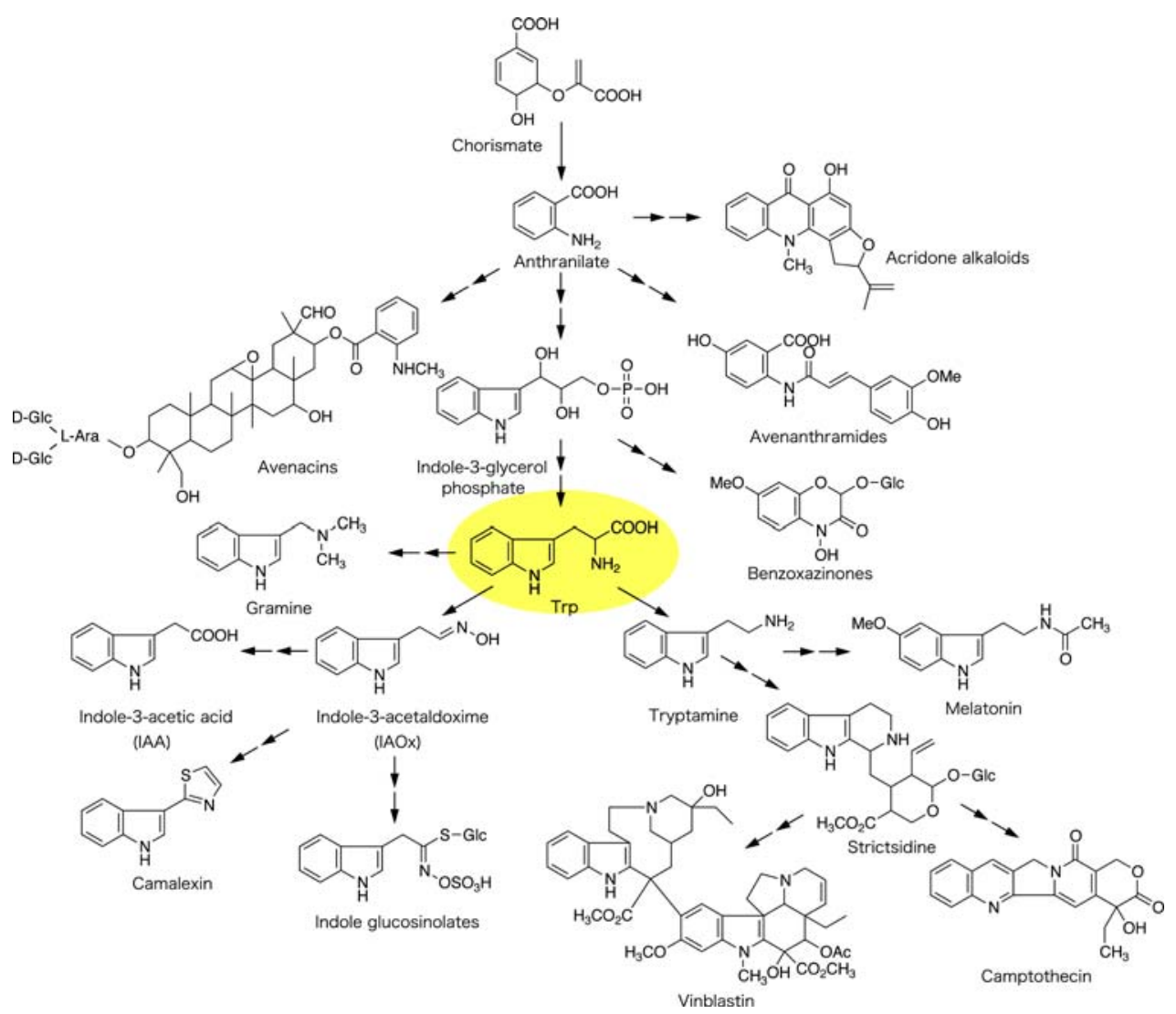


was utilized in the transformation of cultured hairy root of C. roseus to successfully elevate endogenous Trp levels from below detection limits to $2.5 \mathrm{mg} / \mathrm{g}$ dry weight (Hughes et al. 2004). Our group also tried to generate a feedback insensitive AS by introducing a mutation into AS $\alpha$ subunit gene of rice (Tozawa et al. 2001). Based on the single point mutations found in feedback insensitive mutants of Arabidopsis and Salmonella, we designed several mutant genes, including one termed $O A S A 1 D$ which substituted Asp-323 with Asn. This mutation conferred a marked resistance toward 5-methyltryptophan (5MT) when expressed in rice calli under the control of ubiquitin promoter. The AS activity in transformed calli was maintained even in the presence of high concentrations of Trp, and this reduced susceptibility to feedback inhibition gives rise to increases in the level of free $\operatorname{Trp}$ of up to 180-fold. Metabolic flux analysis using ${ }^{13} \mathrm{C}$-labeled Ser as precursor revealed that flux through the Trp biosynthetic pathway was increased about sixfold by the expression of $O A S A 1 D$ in suspensions of cultured rice cells to affect a 45-fold increase in the level of free Trp (Matsuda et al. 2007).

The mutant rice $O A S A 1 D$ gene was also demonstrated to be effective in raising the free Trp level not only in rice plants regenerated from the transformed calli (Tozawa et al. 2001), but also in other plant species, such as potato (Yamada et al. 2004), soybean (S. M. Rahman in preparation), azuki bean (Hanafy et al. 2006), and Arabidopsis (Ishihara et al. 2006). In the case of the regenerated rice plant, overproduction of Trp was stable under field conditions and maintained in seeds and the progenies (Wakasa et al. 2006). The resistance to Trp analogs demonstrated by plants transformed with $O A S A 1 D$ also opened the way to application of the transgene as a selectable marker in gene recombinant experiments (Kawagishi-Kobayashi et al. 2005; Yamada et al. 2004). The structure-catalytic activity relationship of the rice AS protein has now been extensively investigated, using an in vitro transcription-translation system, to reveal the amino acid residues that play an important role in catalytic efficiency and in feedback regulation (Kanno et al. 2004).

The relationship between AS activity and the accumulation of Trp has also been examined in Arabidopsis (Ishihara et al. 2006). Introduction of $O A S A 1 D$ caused an increase in the amounts of Trp varying from 4- to 200-fold among the transformed lines. The transgenic lines had a comparable level of AS activity in the tissues, which were in the same order as those in the untransformed plants. However, the degree of feedback sensitivity of AS activity in the tissue to Trp was different among the transgenic lines, and the observed AS activity in the presence of a specific concentration of Trp correlated well with the amount of free Trp. The result clearly indicated the role of feedback inhibition in controlling the amount of Trp in tissues. The enhancement of Trp accumulation in transgenic plants obtained to date is summarized in Table 1.

\subsection{Metabolic profiling of Trp over-producing plants}

As described above, anthranilate and Trp serve as precursors for a diverse range of nitrogen-containing secondary metabolites (Fig. 2). Thus, the effects of overproduction of Trp on the metabolite profile in plant overexpressing $O A S A 1 D$ are clearly of great interest. Our group first analyzed the profile of aromatic metabolites in $O A S A 1 D$ transgenic rice calli to compare with that of untransformed calli. We used HPLCPDA detection, since alteration of Trp biosynthesis was considered to most likely affect the activity of shikimate pathway and the metabolism of aromatic compounds (Morino et al. 2005). To our surprise, no immediately obvious change in the metabolic profile was observed, except for the intended increase in the amount of Trp. Closer investigation of the OASA1D profile highlighted modest changes in the levels of several minor components. One of these minor components was identified as a novel indole compound, possibly a "deactivated" product of indole glycerol-3-phosphate, an intermediate of the Trp biosynthetic pathway. The role of this compound in rice is unknown.

Rice seeds from field-grown OASA1D transgenic plants also exhibited near-identical aromatic metabolite profiles to that of the wild-type, except for the intended increase of Trp levels (Wakasa et al. 2006). The expected stresses under field conditions caused no unexpected metabolic changes that were associated with activation of Trp biosynthesis. Accumulation of Trp in seeds was, however, accompanied by an increase in the amounts of other free amino acids in contrast to results from transgenic rice calli. The presence of a regulatory mechanism that increased overall amino acid biosynthesis in response to Trp accumulation was speculated analogous to that associated amino acid accumulation in opaque-2 mutants of maize (Wang and Larkins 2001; Wang et al. 2001).

Further metabolic profiling analysis of the whole rice plant revealed an interesting aspect of Trp accumulation in the OASA1D transformant (manuscript in preparation). Thus, the level of Trp in 2- or 4-week-old seedlings was tissue dependent, with the most elevated levels found in the youngest leaf in a plant, followed by lower levels in the leaf sheath and root. Levels in the oldest leaf of the transgenic plant, however, was not significantly different from that of the corresponding leaf in the untransformed plant. Since no difference in AS activity or sensitivity to Trp feedback inhibition was observed among these tissues, it is most likely that active translocation of Trp to actively growing tissues accounts for this tissue dependent distribution. The composition of aromatic components, other 
Table 1 Enhancement of free Trp accumulation by introduction of feedback insensitive AS $\alpha$ genes

\begin{tabular}{|c|c|c|c|c|}
\hline Plant & Introduced gene & Ratio & Note & Literature \\
\hline \multirow[t]{2}{*}{ Rice } & \multirow[t]{2}{*}{ OASA1D } & $42-177$ & Callus & \multirow[t]{2}{*}{ Tozawa et al. (2001) } \\
\hline & & $24-35$ & Leaf & \\
\hline \multirow[t]{2}{*}{ Rice } & \multirow[t]{2}{*}{ OASA1D } & 55.9 & Seed (green house) & \multirow[t]{2}{*}{ Wakasa et al. (2006) } \\
\hline & & 311.0 & Seed (field) & \\
\hline \multirow[t]{3}{*}{ Potato } & \multirow[t]{3}{*}{ OASA1D } & 2.1 & Leaf & \multirow[t]{3}{*}{ Matsuda et al. (2005b) } \\
\hline & & 2.7 & Tuber & \\
\hline & & 23.4 & Microtuber & \\
\hline Rice & OASA2(Y367A/L530D) & $34.6-38.8$ & Callus & Kanno et al. (2005) \\
\hline Arabidopsis & OASA1D & 200 & 2-week-old rosette leaf & Ishihara et al. (2006) \\
\hline Azuki & OASA1D & $6.5-16.5$ & Seed & Hanafy et al. (2006) \\
\hline Soybean & OASA1D & $21.5-50.9$ & Seed & $\begin{array}{l}\text { S. M. Rahman } \\
\text { (in preparation) }\end{array}$ \\
\hline Tobacco & Tabacco feedback insensitive $A S A 2$ & 3 & Youngest expanded leaf & Tsai et al. (2005) \\
\hline Tobacco & Tabacco feedback insensitive $A S A 2$ & 16 & $\begin{array}{l}\text { Leaf } A S A 2 \text { introduced in } \\
\text { the plastide genome }\end{array}$ & Zhang et al. (2001) \\
\hline Astragalus sinicus & Tabacco feedback insensitive $A S A 2$ & $1.3-5.5(8-26)$ & Hairy root culture & Cho et al. $(2000,2004)$ \\
\hline Soybean & Tabacco feedback insensitive $A S A 2$ & $3-6$ & Hairy root culture & Cho et al. (2004) \\
\hline Catharanthus roseus & Arabidopsis mutated $A S A 1$ & 300 & $\begin{array}{l}\text { Hairy root culture (late } \\
\text { exponential stage) } \\
\text { after a 6-day } \\
\text { induction by } \\
\text { dexamethasone }\end{array}$ & Hughes et al. (2004) \\
\hline Maize & Maize mutated $A S A$ & 3.7 & Kernel & Anderson et al. (1997) \\
\hline
\end{tabular}

than changes in Trp levels, in any of the tissues showed no remarkable difference between the transgenic and untransformed plants. Indeed when profiling was performed with an LC-MS platform that allowed detection of more than 1,500 components, differences in metabolite profiles were more significant among tissues than between genotypes. The limited metabolic effect of transformation of rice plant with $O A S A 1 D$ was also demonstrated by transcriptomic analysis which revealed that in only a small number of genes were differentially expressed, albeit modestly (Dubouzet et al. 2007).

The expression of $O A S A 1 D$ in potato also effectively increased the amount of free Trp by 2-20-fold. The HPLCPDA-based profile of aromatic compounds again showed no practical difference between transformed and untransformed plants, except for the intended increase in Trp levels, and no new component was detected as a consequence of transformation (Matsuda et al. 2005b). Further LC-MS-based profiling also revealed no changes either in intact and wounded tissues, suggesting that the transgene effect is limited only to Trp biosynthesis even under stressed conditions (unpublished data).

In transgenic Arabidopsis, accumulation of Trp was accompanied by a slight elevation in the amounts of IGs, possibly due to an increase in substrate supply (Ishihara et al. 2006). Interestingly, the increase in IG contents was more evident when plants were treated with jasmonic acid. The transformation, however, had no significant effect on the induction of the phytoalexin camalexin that is also biosynthesized from Trp in response to the inoculation of a pathogenic fungus.

An LC-MS-based profiling strategy was developed that allowed detection of $\sim 1,400$ components in transformed soybean (S. M. Rahman in preparation). Of these, only a small portion showed any increases in endogenous levels, in addition to the intended increase in Trp. One compound that was elevated, albeit modestly was identified as gamma-glutamyl Trp. Although its biological and physiological role is unknown, it is clearly related to Trp in terms of biosynthesis, and so the increase itself is related to the intended consequences of transformation.

In all cases, the introduction of the OASAID gene into a diverse range of plants appeared to give rise to the intended overproduction of Trp with only minor changes in the entire metabolome. This clearly implies that the biosynthetic conversion of anthranilate to Trp in plants is strictly regulated and proceeds in an orderly manner that effectively utilizes intermediates, even under conditions of increased anthranilate supply due to the expression feedback insensitive AS. Furthermore, it is noteworthy that 
accumulated Trp undergoes no remarkable conversion into any specific catabolites or secondary metabolites. Studies on the subcellular distribution of Trp that contributes to such stability will prove an interesting research subject.

\subsection{Targeted analysis of IAA}

The IAA represents a major natural plant hormone, auxin, and plays an important role in the regulation of plant growth and development. It is generally considered that IAA is biosynthesized from $\operatorname{Trp}$ in plants, although a route that bypasses Trp has also been proposed (Woodward and Bartel 2005). In both cases, anthranilate is commonly considered to be the key precursor. Alteration of AS activity is thus likely to affect the metabolism of IAA. Tissue levels of IAA, however, are usually too low to be monitored by current metabolic profiling techniques and targeted monitoring is therefore required. Absolute quantification of IAA generally requires sensitive detection methods and several specialized procedures based on GC-MS and LC-MS (-MS) have been developed (Chiwocha et al. 2003; Edlund et al. 1995; Kowalczyk and Sandberg 2001; Matsuda et al. 2005a; Ribnicky et al. 1998). Furthermore, precautions are needed to avoid the artifactual formation of IAA during experimental analysis of OASA1D transformants, particularly as high levels of Trp can potentially exacerbate such a problem when dealing with metabolites such as IAA that are present at very low levels.

When assessed in this manner, levels of free IAA in $O A S A 1 D$ transgenic rice calli, rice seeds, and potato have been shown to be higher than in untransformed plants (Matsuda et al. 2005b; Morino et al. 2005; Wakasa et al. 2006) most likely as a consequence of the high-Trp content in these transformants. Measured increases were about twofold in the cases of rice seeds and potato leaf, while that in rice calli ranged from 3- to 60-fold, depending on the transformed lines. Levels of conjugated forms of IAA also appeared to be increased in rice calli and seeds. In spite of these increases, no significant change in morphology or growth rate was observed in any of the transformants. This is in marked contrast to other phenotypically abnormal Arabidopsis mutants characterized by increased IAA levels (Barlier et al. 2000; Boerjan et al. 1995; Zhao et al. 2001). The somewhat lower spikelet fertility and impaired seed germination observed in $O A S A 1 D$ rice seeds may be attributable to the high-Trp levels rather than to increased IAA (Wakasa et al. 2006). In OASAID transformants, compartmentalization of the increased IAA most likely contributes to the minimal changes in the plant growth and development.

\section{What metabolomics of Trp overproducing plants revealed}

6.1 The stability of Trp metabolism

Metabolic profiling of $O A S A 1 D$ plants has demonstrated that the introduction of a feedback-resistant AS has limited effects on other metabolic pathways. In particular, no overaccumulation of secondary metabolites derived from the Trp pathway was observed except for a slight accumulation of a novel indole compound derived from indole glycerol-3-phosphate in rice calli (Morino et al. 2005) and gamma-glutamyl Trp in soybean seeds (S. M. Rahman in preparation).

Two distinct pathways in plants originate from Trp to produce secondary metabolites. The first pathway starts with the decarboxylation of Trp by tryptophan decarboxylase (TDC). Various indole alkaloids are biosynthesized via this pathway. Trp-derived metabolites found in rice are melatonin (Hattori et al. 1995) and tryptamine (Ueno et al. 2003). Tryptamine is the direct product of TDC, while melatonin is probably biosynthesized from tryptamine. The rice genome contains at least one TDC gene orthologous to the TDC gene in C. roseus (Dubouzet et al. 2007). Our group analyzed the contents of indolic amines in transgenic rice expressing $O A S A 1 D$, and found several fold increases in tryptamine and serotonin levels in the rice leaves (Dubouzet et al. 2007). The amount of melatonin was under the detection limit of the LC-MS method used. Given the 200-fold increase in the Trp content in the transformed lines, the observed increase in tryptamine and serotonin levels can be regarded marginal. Thus, funneling of metabolic flow toward secondary metabolism via TDC is strictly regulated at this step.

The first committed reaction in the other Trp-derived pathway is the formation of IAOx by cytochrome P450 enzymes (CYP79B2 and CYP79B3), as mentioned earlier. This pathway has been shown to be involved in the biosynthesis of IGs, IAA (Hull et al. 2000; Mikkelsen et al. 2000; Zhao et al. 2002), and camalexin (Glawischnig et al. 2004) in Arabidopsis. The Arabidopsis seedlings expressing $O A S A 1 D$ accumulated several fold larger amounts of IGs than the wild type (Ishihara et al. 2006). However, the Trp content in the transgenic leaves was over 200-fold greater than that in the wild-type leaves. Thus, metabolic flow into the IG pathway is also under strict regulation.

Sequestration of Trp in a compartment where Trp is not actively metabolized is a plausible mechanism to explain the limited effect of Trp over-accumulation. The presence of a "storage form" of Trp is suggested by flux analysis of Trp biosynthesis, based on the relatively low incorporation of labeled Ser into Trp (Matsuda et al. 2007). It is possible that excess amounts of Trp was transported into the 
vacuole isolating Trp from further metabolism, although the role of the vacuole in amino acid sequestration remains to be clarified. The putative amino acid transporter CAT2 was detected in Arabidopsis tonoplasts when expressed as a GFP fusion protein in transiently transformed protoplasts (Su et al. 2004).

Intermediates in Trp biosynthesis also play a role as precursors for secondary metabolism. However, metabolic profiling of transgenic plants expressing $O A S A 1 D$ demonstrated that the amounts of those compounds are quite low. The levels of anthranilate in the transgenic rice plants expressing $O A S A 1 D$ were only several times greater than that in the wild type (Matsuda et al., in preparation) in marked contrast to the enhanced accumulation of Trp. The large capacity for the Trp synthesis suggests that the accumulation of intermediates may be unfavorable in the wild-type and transformed plants.

The highly fluorescent Arabidopsis Trp-requiring mutant trp1-1 is impaired in the second step of Trp synthesis (Last and Fink 1988). The mutant cannot utilize anthranilate for Trp synthesis, and accumulates the excess as anthranilate glucoside. The glucosyltransferase gene responsible for glucosylation of anthranilate has been isolated (Quiel and Bender 2003). Although the toxicity of anthranilate to plants is unclear, it seems likely that glucosylation of anthranilate functions to eliminate any potential deleterious effects. However, in the OASAID expressing Arabidopsis, the anthranilate glucoside was not detected. Thus, the glucosylation mechanism seems less important than the rapid conversion of anthranilate into Trp in plants not impaired in Trp synthesis.

\subsection{Interaction between the pathways for Trp and Phe}

The Trp is derived from the shikimate pathway in common with Phe and Tyr. Yet knowledge on the interaction of the biosynthetic pathways for these aromatic amino acids remains limited. It is possible to assume two modes of interaction between the two distinct pathways.

The first mode of interaction involves competition for substrate supply. The first committed reactions in the two pathways are catalyzed by $\mathrm{AS}$ and chorismate mutase (CM), respectively. Both enzymes use chorismate as the substrate. Thus, the activation of one pathway may cause suppression of the other. Whilst, the Phe and Tyr pathway provides these aromatic amino acids for protein synthesis, Phe also functions as a precursor of numerous secondary metabolites including phenylpropanoids and flavonoids. Further, Phe is a precursor of lignin, one of the major cell wall constituents. Thus, metabolic flux in this pathway can be predicted to be much larger than that in the Trp pathway. Accordingly, activation of the Trp pathway should have minimal impact on the Phe pathway. However, the actual level of Trp in rosette leaves of OASA1D Arabidopsis amounted to 3,000 nmol/gFW, which represented a considerable amount relative to phenylpropanoids (Ishihara et al. 2006). This level was much higher than those of the major secondary metabolites detected in the rosette leaves.

A second potential mode of interaction is feedback regulation of $\mathrm{CM}$ by Trp. All plants investigated to date have at least two isoforms of CM; one isoform localized in the cytosol and the other in the plastids (Romero et al. 1995). The isoforms in the plastids have been demonstrated to be activated by Trp (Eberhard et al. 1996; Singh et al. 1986). Thus, over-accumulation of Trp may activate CM, leading to an increase in levels of Phe and Tyr in OASA1D transgenic plants.

Seedlings of Arabidopsis contain a variety of Phederived secondary metabolites such as phenylpropanoids (e.g., sinapoyl malate and synapoyl glucose) and flavonoids (kaempferol glucosides and quercetin glucosides). In an analysis of metabolic changes in Arabidopsis expressing $O A S A 1 D$, it was found that levels of phenylpropanoids and flavonoids decreased in those lines that accumulated high concentrations of Trp (Ishihara et al. 2006). This was at first thought to be a manifestation of competition between the Trp and Phe pathways. To evaluate this hypothesis, Phe and Tyr levels in the transgenic plants were assessed. Unexpectedly, levels of Phe and Tyr were found to be high in the lines that accumulated Trp at high concentrations in comparison with their levels in the wild type plant. The increase in levels of Phe and Tyr is likely to be attributed to the feedback activation of CM by Trp. Thus, the observed decrease in levels of Phe-derived secondary metabolites may be due to the suppression of the introduction of carbon into that pathway. Since a decrease was found in all of the identified Phe-derived secondary metabolites, the site of regulation was probably at the early $\operatorname{step}(\mathrm{s})$ in the phenylpropanoid pathway. If there is a mechanism for the suppression of the phenylpropanoid pathway in response to Trp accumulation, it may also provide a clue to why Phe levels were raised. Analysis of the gene expression, measurement of enzyme activity and analysis of metabolic flux of the phenylpropanoid pathway will prove informative in revealing further details of metabolic changes in $O A S A 1 D$ expressing Arabidopsis.

No decreases in the levels of Phe-derived secondary metabolites were observed in potato tuber and leaf expressing OASA1D (Matsuda et al. 2005b). This may be because of the relatively small increase in Trp content in potato in comparison with Arabidopsis (see Table 1).

Interaction between the Trp and Phe/Tyr pathways has been suggested by the rice mutant $m t r l$ that is resistant to the Trp analog, 5MT (T. Yamada et al., 2007, submitted data). The mutant calli contained markedly elevated levels of $\gamma$-glutamylphenylalanine and multiple Phe-derived 
secondary metabolites including glucosylbenzyl alcohol, feruloylquinate, and rosine glucosides. The presence of these metabolites suggested alternations in the Phe metabolism in this mutant that differed from previously isolated 5MT resistant lines that had mutations in enzymes directly associated with the Trp pathway. Positional cloning of the mutated gene mtrl revealed that a single base substitution in the arogenate dehydratase gene is responsible for the mutant phenotype. This enzyme catalyzes the final step in Phe synthesis and is controlled by feedback inhibition by Phe. The replacement of a single amino acid in the mutated enzyme causes relaxed feedback sensitivity of the enzyme. Metabolic profiling of the calli expressing the mutated gene revealed that they contained elevated levels of Phe and Trp. The elevated levels of Trp explained the increased resistance of the mutant against 5MT although the linkage between the arogenate dehydratase mutation and Trp accumulation has not been elucidated.

\subsection{Translocation of Trp in plants}

Analysis of Trp distribution in OASA1D rice seedlings showed accumulation of Trp occurred at highest concentrations in newly formed leaves (Matsuda et al., in preparation). A similar trend was observed in the OASA1D Arabidopsis (Ishihara et al. 2006). Thus, the rosette leaves and roots of 2-week-old seedlings accumulated Trp at high concentrations, whilst the Trp concentrations in the rosette leaves and roots in 5-week-old seedlings were similar to those in the wild type plant. In contrast, the silique and flowers of the 5-week-old seedlings contained high concentrations of Trp. These Trp distribution patterns in rice and Arabidopsis seedlings expressing OASAID suggested translocation of additional amounts of Trp from source organs to the sink organs. The Trp content in the siliques and flowers in the 5-week-old OASAID seedlings were 65and 135-fold greater, respectively, than those in the wild type. These increased levels were similar to those in the rosette leaves of 2-week-old seedlings (200-fold), suggesting that the plant had the capacity to translocate excess Trp from source organs to reproductive organs. This highcapacity translocation system may represent a mechanism for the efficient utilization of assimilated metabolites for reproduction.

In the $O A S A 1 D$ expressing plants, any excess Trp that is not required by the source organs is probably transported by some translocation system to the sink. The translocation system of amino acids, however, is not completely been understood. The Arabidopsis genome contains more than 50 putative amino acid transporter genes (Liu and Bush 2006), which includes ANTl that does transport Trp (Chen et al. 2001). However, the individual function of each of these putative transporters remains unclear. This can be attributed to a lack of distinct phenotypic changes in lossof-function mutants of these amino acid transporter genes, most probably due to some form of functional redundancy. Since the OASAID plants appear to transfer much larger amounts of Trp relative to the wild type, it may be possible to detect alterations in amino acid composition between the sources and sink organs by the introduction of loss-offunction mutation in the amino acid transporter genes in the $O A S A 1 D$ background. The analysis of these mutants could be a new approach for in vivo functional characterization of amino acid transporters.

\section{Concluding remarks}

Overproduction of Trp in multiple plant species expressing $O A S A 1 D$ has clearly demonstrated the importance of feedback regulation in the control of Trp pool sizes. Feedback resistant AS $\alpha$ subunits have been found in other sources, such as tobacco cell cultures (Song et al. 1998), Arabidopsis trp5 mutant (Kreps et al. 1996; Li and Last 1996), and R. graveolens (Bohlmann et al. 1996). However, the increased levels of Trp content in the plants expressing these AS $\alpha$ genes are not as large as those in the OASA1D expressing plants ( $\mathrm{Li}$ and Last 1996, Tsai et al. 2005). This difference is likely attributable to the lower feedback sensitivity of OASA1D than other feedback resistant AS $\alpha$ subunits because total AS activity is not increased by the expression of OASA1D. However, OASA1D still exhibits slight sensitivity to high concentrations of Trp. It may thus be possible to develop an AS $\alpha$ enzyme that is more resistant to Trp by further introduction of mutations in $O A S A 1 D$. Recently, rational modification of the rice $O A S A 2$ gene was conducted based on structural factors associated with the catalytic function and feedback regulation of the enzyme molecule. An improved enzyme both in terms of catalytic activity and tolerance to feedback inhibition by $\operatorname{Trp}$ was thus successfully obtained, and was effective in elevating the levels of free Trp in rice calli (Kanno et al. 2005). Other plants expressing this highly modified enzyme should exhibit more pronounced accumulation of Trp.

Feedback regulation of AS has been characterized in many plants including both dicotyledonous and monocotyledonous species. Given the fact that even a single point mutations in the AS $\alpha$ gene causes the relaxed feedback sensitivity yet many plants actually retain feedback sensitive AS $\alpha$, this feedback regulation should be important for the life of individuals and species. However, no significant negative effects of the introduction of $O A S A 1 D$ on plant growth and development have been observed; factors related to reproduction may be influenced by accumulation of Trp, however. Indeed, some rice lines that express 
OASAID showed a reduced rate of spikelet fertility and yield although any logical connection between these traits and Trp accumulation has not been clarified (Wakasa et al. 2006). In addition, stress conditions, for example, lownitrogen supply or other elements could, at least in principle, more strongly affect physiology in OASAID expressing plants as the production of excess amounts of Trp could be considered wasteful. Thus, OASA1D transgenic plants could provide a unique opportunity to elucidate the physiological significance of feedback regulation in the life cycle of the plants.

The Trp overproduction had a limited effect on downstream metabolism in investigated species, indicating that the metabolic flow from Trp into secondary metabolism is strictly regulated. From the viewpoint of safety evaluations of metabolically engineered crops this is a preferred characteristic and highlights the value of the Trp biosynthetic pathway as a target. On the other hand, some Trp-derived secondary metabolites are pharmaceutically useful and others play significant roles in plant physiology and in interactions with the plant's environment.

In summary, by genetic modification of downstream enzymatic steps, the control of the production of Trpderived secondary metabolites has been achieved. The introduction of feedback resistant AS $\alpha$ will be an approach to enhance the productivity of secondary metabolism in combination with the modification of downstream enzymatic steps.

Acknowledgments This work was supported by CREST of the Japan Science and Technology Agency (JST).

\section{References}

Anderson, P. C., Chomet, P. S., \& Griffor, M. C. (1997). Anthranilate synthase gene and use thereof. Patent: WO 9726366.

Bak, S., Tax, F. E., Feldmann, K. A., Galbraith, D. W., \& Feyereisen, R. (2001). CYP83B1, a cytochrome P450 at the metabolic branch point in auxin and indole glucosinolate biosynthesis in Arabidopsis. Plant Cell, 13, 101-111.

Baker, J. M., Hawkins, N. D., Ward, J. L., Lovegrove, A., Napier, J. A., Shewry, P. R., Beale, M. H. (2006). A metabolomic study of substantial equivalence of field-grown genetically modified wheat. Plant Biotechnology Journal, 4, 381-392.

Bamba, T., \& Fukusaki, E. (2006). Technical problems and practical operations in plant metabolomics. Journal of Pesticides Science, 31, 300-304.

Barlier, I., Kowalczyk, M., Marchant, A., Ljung, K., Bhalerao, R., Bennett, M., Sandberg, G., \& Bellini, C. (2000). The SUR2 gene of Arabidopsis thaliana encodes the cytochrome P450 CYP83P1, a modulator of auxin homeostasis. Proceedings of the National Academy of Sciences of the United States of America, 97, 14819-14824.

Belser, W. L., Baron Murphy, J., Delmer, D. P., \& Mills, S. E. (1971). End product control of Trp biosynthesis in extracts and intact cells of the higher plant Nicotiana tabacum var. Wisconsin 38 . Biochimica et Biophysica Acta, 237, 1-10.
Beyer, P., Al-Babili, S., Ye, X., Lucca, P., Schaub, P., Welsch, R., \& Potrykus, I. (2002). Golden rice: Introducing the $\beta$-carotene biosynthesis pathway into rice endosperm by genetic engineering to defeat vitamin A deficiency. The Journal of Nutrition, 132, $506 \mathrm{~S}-510 \mathrm{~S}$.

Boerjan, W., Cervera, M.-T., Delarue, M., Beckman, T., Dewitte, W., Bellini, C., Caboche, M., Van Onckelen, H., Van Montagu, M., \& Inzé, D. (1995). Superroot, a recessive mutation in Arabidopsis, confers auxin overproduction. Plant Cell, 7, 1405-1419.

Bohlmann, J., DeLuca, V., Eilert, U., \& Martin, W. (1995). Purification and cDNA cloning of anthranilate synthase from Ruta graveolens: Modes of expression and properties of native and recombinant enzymes. Plant Journal, 7, 491-501.

Bohlmann, J., Lins, T., Martin, W., \& Eilert, U. (1996). Anthranilate synthase from Ruta graveolens. Plant Physiology, 111, 507-514.

Boudet, A. M., \& Grima-Pettenati, J. (1996). Lignin genetic engineering. Molecular Breeding, 2, 25-39.

Chavadej, S., Brisson, N., McNeil, J. N., \& De Luca, V. (1994). Redirection of tryptophan leads to production of low indole glucosinolate canola. Proceedings of the National Academy of Sciences of the United States of America, 91, 2166-2170.

Chen, L., Ortiz-Lopez, A., Jung, A., \& Bush, D. R. (2001) ANT1, an aromatic and neutral amino acid transporter in Arabidopsis. Plant Physiology, 125, 1813-1820.

Chiwocha, S. D. S., Abrams, S. R., Ambrose, S. J., Cutler, A., Loewen, M., Ross, A. R. S., \& Kermode, A. R. (2003). A method for profiling classes of plant hormones and their metabolites using liquid chromatography-electrospray ionization tandem mass spectrometry: And analysis of hormone regulation of thermodormancy of lettuce (Lactuca sativa L.) seeds. Plant Journal, 35, 405-417.

Cho, H.-J., Brotherton, J. E., Song, H. -S., \& Widholm, J. M. (2000). Increasing tryptophan synthesis in a forage legume Astragalus sinicus by expressing the tobacco feedback-insensitive anthranilate synthase (ASA2) gene. Plant Physiology, 123, 1069-1076.

Cho, H.-J., Brotherton, J. E., \& Widholm, J. M. (2004). Use of tobacco feedback-insensitive anthranilate synthase gene (ASA2) as a selectable marker for legume hairy root transformation. Plant Cell Report, 23, 104-113.

Choi, H.-K., Choi, Y. H., Verberne, M., Lefeber, A. W. M., Erkelens, C., \& Verpoorte, R. (2004). Metabolic fingerprinting of wild type and transgenic tobacco plants by ${ }^{1} \mathrm{H}$ NMR and multivariate analysis technique. Phytochemistry, 65, 857-864.

Defernez, M., Gunning, Y. M., Parr, A. J., Shepherd, L. T., Davies, H. V., \& Colquhoun, I. J. (2004). NMR and HPLC-UV profiling of potatoes with genetic modifications to metabolic pathways. Journal of Agricultural and Food Chemistry, 52, 6075-6085.

Delarue, M., Prinsen, E., Onckelen, H. V., Caboche, M., \& Bellini, C. (1998). Sur2 mutations of Arabidopsis thaliana define a new locus involved in the control of auxin homeostasis. Plant Journal, 14, 603-611.

Dewaele, E., Craciun, A., Vauterin, M., Frankard, V., Suharyanto, E., Tadesse, J., \& Jacobs, M. (2002). Metabolic engineering of a complex biochemical pathway: The lysine and threonine biosynthesis as an example. Phytochemistry Review, 1, 125-133.

Dill, G. M. (2005). Glyphosate-resistant crops: History, status and future. Pest Management Science, 61, 219-224.

Dubouzet, J. G., Ishihara, A., Matsuda, F., Miyagawa, H., Iwata, H., Wakasa, K. (2007). Integrated microarray-metabolic analysis of high-tryptophan rice expressing a mutant anthranilate synthase alpha subunit. Journal of Experimental Botony (in press).

Eberhard, J., Ehrler, T., Epple, P., Felix, G., Raesecke, H.-R., Amrheim, N., \& Schmid, J. (1996). Cytosolic and plastidic chorismate mutase isozymes from Arabidopsis thaliana: Molecular characterization and enzymatic properties. Plant Journal, $10,815-821$. 
Edlund, A., Eklof, S., Sundberg, B., Moritz, T., \& Sandberg, G. (1995). A microscale technique for gas chromatography-mass spectrometry measurements of picogram amounts of indole-3acetic acid in plant tissues. Plant Physiology, 108, 1043-1047.

Fiehn, O., Kopka, J., Dörmann, P., Altmann, T., Trethewey, R. N., \& Willmitzer, L. (2000). Metabolite profiling for plant functional genomics. Nature Biotechnology, 18, 1157-1161.

Fraser, P. D., Pinto, M. E. S., Holloway, D. E., \& Bramley, P. M. (2000). Application of high-performance liquid chromatography with photodiode array detection to the metabolic profiling of plant isoprenoids. Plant Journal, 24, 551-558.

Frey, M., Chomet, P., Glawischnig, E., Stettner, C., Grün, S., Winklmair, A., Eisenreich, W., Bacher, A., Meeley, R. B., Briggs, S. P., Simcox, K., \& Gierl A. (1997). Analysis of a chemical plant defense mechanism in grasses. Science, 277, 696-699.

Galili, G., Amir, R., Hoefgen, R., \& Hesse, H. (2005). Improving the levels of essential amino acids and sulfur metabolites in plants. Biological Chemistry, 386, 817-831.

Galili, G., \& Höfgen, R. (2002). Metabolic engineering of amino acids and storage proteins in plants. Metabolic Engineering, 4, 3-11.

Gall, G. L., Colquhoun, I. J., Davis, A. L., Collins, G. J., \& Verhoeyen, M. E. (2003). Metabolite profiling of tomato (Lycopersicon esculentum) using ${ }^{1} \mathrm{H}$ NMR spectroscopy as a tool to detect potential unintended effects following a genetic modification. Journal of Agricultural and Food Chemistry, 51, $2447-2456$.

Garrat, L. C., Linforth, R., Taylor, A. J., Lowe, K. C., Power J. B., \& Davey, M. R. (2005). Metabolite fingerprinting in transgenic lettuce. Plant Biotechnology Journal, 3, 165-174.

Glawischnig, E., Hansen, B. G., Olsen, C. E., \& Halkier, B. A. (2004). Camalexin is synthesized from indole-3-acetoaldoxime, a key branching point between primary and secondary metabolism in Arabidopsis. Proceedings of the National Academy of Sciences of the United States of America, 101, 8245-8250.

Gröger, D., \& Johne, S. (1968). Zur Biosynthesise einiger Alkaloide von Glycosmis arborea (Rutaceae). Zeitschrift fur Naturforschung, 23b, 1072-1075.

Hanafy, M. S., Rahman, S. M., Khalafalla, M. M., El-Shemy, H. A., Nakamoto, Y., Ishimoto, M., \& Wakasa, K. (2006). Accumulation of free tryptophan in azuki bean (Vigna angularis) induced by expression of a gene $(O A S A 1 D)$ for a modified alpha-subunit of rice anthranilate synthase. Plant Science, 171, 670-676.

Hattori, A., Migitaka, H., Iigo, M., Itoh, M., Yamamoto, K., OhtaniKaneko, R., Hara, M., Suzuki, T., \& Reiter, R. J. (1995). Identification of melatonin in plants and its effects on plasma melatonin levels and binding to melatonin receptors in vertebrates. Biochemstry and Molecular Biology International, 35, 627-634.

Hemm, M. R., Ruegger, M. O., \& Chapple, C. (2003). The Arabidopsis ref2 mutant is defective in the gene encoding CYP83A1 and shows both phenylpropanoid and glucosinolate phenotypes. Plant Cell, 15, 179-194.

Hirai, M. Y., Yano, M., Goodenowe, D. B., Kanaya, S., Kimura, T, Awazuhara, M., Arita, M, Fujiwara, T., \& Saito, K. (2004). Integration of transcriptomics and metabolomics for understanding of global responses to nutritional stresses in Arabidopsis thaliana. Proceedings of the National Academy of Sciences of the United States of America, 101, 10205-10210.

Hossain, T., Rosenberg, I., Selhub, J., Kishore, G., Beachy, G., \& Schubert, K. (2004). Enhancement of folates in plants through metabolic engineering. Proceedings of the National Academy of Sciences of the United States of America, 101, 5158-5163.

Hughes, E. K., Hong, S.-B., Gibson, S. I., Shanks, J. V., \& San, K.-Y. (2004). Expression of a feedback-resistant anthranilate synthase in Catharanthus roseus hairy roots provides evidence for tight regulation of terpenoid indole alkaloid levels. Biotechnology and Bioengineering, 86, 718-727.

Huhman, D. V., \& Sumner, L. W. (2002). Metabolic profiling of saponins in Medicago sativa and Medicago truncatula using HPLC coupled to an electrospray ion-trap mass spectrometer. Phytochemistry, 59, 347-360.

Hull, A. K., Vij, R., \& Celenza, J. L. (2000). Arabidopsis cytochrome P450s that catalyze the first step of tryptophan-dependent indole3 -acetic acid biosynthesis. Proceedings of the National Academy of Sciences of the United States of America, 97, 2379-2384.

Ikeda, M. (2006). Towards bacterial strains overproducing L-tryptophan and other aromatics by metabolic engineering. Applied Microbiology and Biotechnology, 69, 615-626.

Ishihara, A., Ohtsu, Y., \& Iwamura, H. (1999). Biosynthesis of oat avenanthramide phytoalexins. Phytochemistry, 50, 237-242.

Ishihara, A., Asada, Y., Takahashi, Y., Yabe, N., Komeda, Y., Nishioka, T., Miyagawa, H., \& Wakasa, K. (2006). Metabolic changes in Arabidopsis thaliana expressing the feedback-resistant anthranilate synthase $\alpha$-subunit gene OASA1D. Phytochemistry, 67, 2349-2362.

Kanno, T., Kasai, K., Ikejiri-Kannno, Y., Wakasa, K., \& Tozawa, Y. (2004). In vitro reconstitution of rice anthranilate synthase: Distinct functional properties of the $\alpha$-subunits OASA1 and OASA2. Plant Molecular Biology, 54, 11-23.

Kanno, T., Komatsu, A., Kasai, K., Dubouzet, J. G., Sakurai, M., Ikejiri-Kanno, Y., Wakasa, K., \& Tozawa, Y. (2005). Structurebased in vitro engineering of the anthranilate synthase, a metabolic key enzyme in the plant Trp pathway. Plant Physiology, 138, 2260-2268.

Kawagishi-Kobayashi, M., Yabe, N., Tsuchiya, M., Harada, S., Kobayashi, T., Komeda, Y., \& Wakasa, K. (2005). Rice $O A S A 1 D$, a mutant anthranilate synthase $\alpha$ subunit gene, is an effective selectable marker for transformation of Arabidpsis thaliana. Plant Biotechnology, 22, 271-276.

Kowalczyk, M., \& Sandberg, G. (2001). Quantitative analysis of indole-3-acetic acid metabolites in Arabidopsis. Plant Physiology, 127, 1845-1853.

Kreps, J. A, Ponappa, T., Dong, W., \& Town, C. D. (1996). Molecular basis of $\alpha$-methyltryptophan resistance in amt-1, a mutant of Arabidopsis thaliana with altered tryptophan metabolism. Plant Physiology, 110, 1159-1165.

Last, R. L., \& Fink, G. R. (1988). Tryptophan-requiring mutants of the plant Arabidpsis thaliana. Science, 240, 205-240.

Li, J., \& Last, R. L. (1996). The Arabidopsis thaliana trp5 mutant has a feedback-resistant anthranilate synthase and elevated soluble tryptophan. Plant Physiology, 110, 51-59.

Liu, X., \& Bush, D. R. (2006). Expression and transcriptional regulation of amino acid transporters in plants. Amino Acids, 30, 113-120.

Manetti, C., Bianchetti, C., Bizzarri, M., Casciani, L., Castro, C., D'Ascenzo, G., Delfini, M., Di Cocco, M. E., Laganà, A., Miccheli, A., Motto, M., \& Conti, F. (2004). NMR-based metabonomic study of transgenic maize. Phytochemistry, 65, 3187-3198.

Matsuda, F., Miyazawa, H., Wakasa, K., \& Miyagawa, H. (2005a). Quantification of indole-3-acetic acid and amino acid conjugates in rice by liquid chromatography-Electrospray ionizationTandem mass spectroscopy. Bioscience Biotechnology and Biochemistry, 69, 778-783.

Matsuda, F., Wakasa, K., \& Miyagawa, H. (2007). Metabolic flux analysis in plants using dynamic labeling technique: Application to tryptophan biosynthesis in cultured rice cells. Phytochemistry, doi: 10.1016/j.phytochem.2007.03.031.

Matsuda, F., Yamada, T., Miyazawa, H., Miyagawa, H., \& Wakasa, K. (2005b). Characterization of tryptophan-overaccumulating 
potato transgenic for a mutant rice anthranilate synthase a-subunit gene (OASA1D). Planta, 222, 535-545.

Matsukawa, T., Ishihara, A., \& Iwamura, H. (2002). Induction of anthranilate synthase activity by elicitors in oats. Zeitschrift fur Naturforschung, 57c, 121-128.

Matsuoka, M., Furbank, R. T., Fukuyama, H., \& Miyao, M. (2001). Molecular engineering of $\mathrm{C}_{4}$ photosynthesis. Annual Review of Plant Physiology and Plant Molecular Biology, 52, 297-314.

Mayer, M. J., Narbad, A., Parr, A. J., Parker, M. L., Walton, N. J., Mellon, F. A., \& Michael, A. J. (2001). Rerouting the plant phenylpropanoid pathway by expression of a novel bacterial enoyl-CoA hydratase/lyase enzyme function. Plant Cell, 13, $1669-1682$.

Mikkelsen, M. D., Hansen, C. H., Wittstock, U., \& Halkier, B. A. (2000). Cytochrome P450 CYP79B2 from Arabidopsis catalyzes the conversion of tryptophan to indole-3-acetaldoxime, a precursor of indole glucosinolate and indole-3-acetic acid. Journal of Biological Chemistry, 275, 33712-33717.

Mikkelsen, M. D., Naur, P., \& Halkier, B. A. (2004). Arabidopsis mutants in the $\mathrm{C}-\mathrm{S}$ lyase of glucosinolate biosynthesis establish a critical role for indole-3-acetaldoxime in auxin homeostasis. Plant Journal, 37, 770-777.

Morino, K., Matsuda, F., Miyazawa, H., Sukegawa, A., Miyagawa, H., \& Wakasa, K. (2005). Metabolic profiling of tryptophanoverproducing rice calli that express a feedback-insensitive alpha subunit of anthranilate synthase. Plant Cell Physiology, 46, $514-521$.

Morollo, A. A., \& Eck, M. J. (2001). Structure of the cooperative allosteric anthranilate synthase from Salmonella typhimurium. Nature Structural Biology, 8, 243-247.

Morgenthal, K., Weckwerth, W., \& Steuer, R. (2006). Metabolomic networks in plants: Transitions from pattern recognition to biological interpretation. Biosystems, 83, 108-117.

Murooka, Y., Mori, Y., \& Hayashi, M. (2002). Variation of the amino acid content of Arabidopsis seeds by expressing soybean aspartate aminotranferase gene. Journal of Bioscience and Bioengineering, 94, 225-230.

Niyogi, K. K., \& Fink, G. R. (1992). Two anthranilate synthase genes in Arabidopsis: Defense-related regulation of the tryptophan pathway. Plant Cell, 4, 721-733.

Niyogi, K. K., Last, R. L., Fink, G. R., \& Keith, B. (1993). Suppressors of trpl fluorescence identify a new Arabidopsis gene, $\operatorname{trp} 4$, encoding the anthranilate synthase $\beta$ subunit. Plant Cell, 5, 1011-1027.

Nomura, T., Ishihara, A., Imaishi, H., Endo, T. R., Ohkawa, H., \& Iwamura, H. (2002). Molecular characterization and chromosomal localization of cytochrome P450 genes involved in the biosynthesis of cyclic hydroxamic acids in hexaploid wheat. Molecular Genetics and Genomics, 267, 210-217.

Nunes-Nesi, A., Carrari, F., Lytovchenko, A., \& Fernie, A. R. (2005). Enhancing crop yield in Solanaceous species through the genetic manipulation of energy metabolism. Biochemical Society Transactions, 33, 1430-1434.

Oikawa, A., Nakamura, Y., Ogura, T., Kimura, A., Suzuki, H., Sakurai, N., Shinbo, Y., Shibata, D., Kanaya, S., \& Ohta, D. (2006). Clarification of pathway-specific inhibition by Fourier transform ion cyclotron resonance/mass spectrometry-based metabolic phenotyping studies. Plant Physiology, 142, 398-413.

O'Donovan, D., \& Leete, E. (1963). Biosynthesis of gramine: Feeding experiments with tryptophan- $\beta-\left[\mathrm{H}^{3}, \mathrm{C}^{14}\right]$. Journal of the American Chemical Society, 85, 461-463.

Parry, M. A. J., Madgwick, P. J., Carvalho, J. F. C., \& Andralojc, P. J. (2007). Prospects for increasing photosynthesis by overcoming the limitations of Rubisco. Journal of Agricultural Science, 145, $31-43$.
Poulsen, C., Bongaerts, R. J. M., \& Verpoorte, R. (1993). Purification and characterization of anthranilate synthase from Catharanthus roseus. European Journal of Biochemistry, 212, 431-440.

Quiel, J. A., \& Bender, J. (2003) Glucose conjugation of anthranilate by the Arabidpsis UGT74F2 glucosyltransferase is required for tryptophan mutant blue fluorescence. Journal of Biological Chemistry, 278, 6275-6281.

Ribnicky, D. M., Cooke, T. J., \& Cohen, J. D. (1998). A microtechnique for the analysis of free and conjugated indole-3-acetic acid in milligram amounts of plant tissue using a benchtop gas chromatograph-mass spectrometer. Planta, 204, 1-7.

Roessner-Tunali, U., Hegemann, B., Lytovchenko, A., Carrari, F., Bruedigam, C., Granot, D., \& Fernie, A. R. (2003). Metabolic profiling of transgenic tomato plants overexpressing hexokinase reveals that the influence of hexose phosphorylation diminishes during fruit development. Plant Physiology, 133, 84-99.

Rohde, A., Morreel, K., Ralph, J., Goeminne, G., Hostyn, V., De Rycke, R., Kushnir, S., Van Doorsselaere, J., Joseleau, J.-P., Vuylsteke, M., Van Driessche, G., Van Beeumen, J., Messens, E., \& Boerjan, W. (2004). Molecular phenotyping of the pall and pal2 mutants of Arabidopsis thaliana reveals far-reaching consequences on phenylpropanoid, amino acid, and carbohydrate metabolism. Plant Cell, 16, 2749-2771.

Romero, R. M., Roberts, M. F., \& Phillipson, J. D. (1995). Chorismate mutase in microorganisms and plants. Phytochemistry, 40, 1015-1025.

Saruul, P., Srienc, F., Somers, D. A., \& Samac, D. A. (2002). Production of a biodegradable plastic polymer, poly-betahydroxybutyrate in transgenic alfalfa. Crop Science, 42, 919-927.

Shelton, A. M., Tang, J. D., Roush, R. T., Metz, T. D., \& Earle, E. D. (2000). Field tests on managing resistance to Bt-engineered plants. Nature Biotechnology, 18, 339-342.

Singh, B. K., Lonergan, S. G., \& Conn, E. E. (1986). Chorismate mutase isoenzymes from selected plants and their immunological comparison with the isoenzymes from Sorghum bicolor. Plant Physiology, 81, 717-722.

Song, H.-S., Brotherton, J. E., Gonzales, R. A., \& Widholm, J. M. (1998). Tissue culture-specific expression of a naturally occurring tobacco feedback-insensitive anthranilate synthase. Plant Physiology, 117, 533-543.

Spraggon, G., Kim, C., Nguyen-Huu, X., Yee, M. -C, Yanofsky, C., \& Mills, S. E. (2001). The structures of anthranilate synthase of Serratia marcescens crystallized in the presence of $(i)$ its substrates, chorismate and glutamate, and a product, glutamate, and (ii) its end-product inhibitor, L-tryptophan. Proceedings of the National Academy of Sciences of the United States of America, 98, 6021-6026.

Stein, A. J., Sachdev, H. P. S., \& Qaim, M. (2006). Potential impact and cost-effectiveness of Golden Rice. Nature Biotechnology, 24, 1200-1201.

Su, Y.-H., Frommer, W. B., \& Ludewig, U. (2004). Molecular and functional characterization of a family of amino acid transporter from Arabidopsis. Plant Physiology, 136, 3104-3113.

Taylor, J., King, R. D., \& Altmann, T., Fiehn, O. (2002). Application of metabolomics to plant genotype discrimination using statistics and machine learning. Bioinformatics, 18, S241-S248.

Thelen, J. J., \& Ohlrogge, J. B. (2002). Metabolic engineering of fatty acid biosynthesis in plants. Metabolic Engineering, 4, 12-21.

Tozawa, Y., Hasegawa, H., Terakawa, T., \& Wakasa, K. (2001). Characterization of rice anthranilate synthase a-subunit genes $O A S A 1$ and $O A S A 2$. Tryptophan accumulation in transgenic rice expressing a feedback-insensitive mutant of OASA1. Plant Physiology, 126, 1493-1506. 
Tsai, F.-Y., Brotherton, J. E., \& Widholm, J. M. (2005). Overexpression of the feedback-insensitive anthranilate synthase gene in tobacco causes tryptophan accumulation. Plant Cell Report, 23, 548-556.

Ueno, M., Shibata, H., Kihara, J., Honda, Y., \& Arase, S. (2003). Increased tryptophan decarboxylase and monoamine oxidase activities induce Sekiguchi lesion formation in rice infected with Magnaporthe grisea. Plant Journal, 36, 215-228.

von Roepenack-Lahaye, E., Degenkolb, T., Zerjeski, M., Franz, M., Roth, U., Wessjohann, L., Schmidt, J., Scheel, D., \& Clemens, S. (2004). Profiling of Arabidopsis secondary metabolites by capillary liquid chromatography coupled to electrospray ionization quadrupole time-of-flight mass spectrometry. Plant Physiology, 134, 548-559.

Wakasa, K., Hasegawa, H., Nemoto, H., Matsuda, F., Miyazawa, H., Tozawa, Y., Morino, K., Komatsu, A., Yamada, T., Terakawa, T., \& Miyagawa, H. (2006). High-level tryptophan accumulation in seeds of transgenic rice and its limited effects on agronomic traits and seed metabolite profile. Journal of Experimental Botony, 57, 3069-3078.

Wang, X., \& Larkins, A. (2001). Genetic analysis of amino acid accumulation in opaque-2 maize endosperm. Plant Physiology, $125,1766-1777$.

Wang, X., Stumpf, D. K., \& Larkins, A. (2001). Aspartate kinase 2. A candidate gene of quantitative trait locus influencing free amino acid content in maize endosperm. Plant Physiology, 125, 17781787.

Ward, J. L., Baker, J. M., \& Beale, M. H. (2007). Recent application of NMR spectroscopy in plant metabolomics. FEBS Journal, $274,1126-1131$

Weckwerth, W., Loureiro, M. E., Wenzel, K., \& Fiehn, O. (2004). Differential metabolic networks unravel the effects of silent plant phenotypes. Proceedings of the National Academy of Sciences of the United Stats of America, 101, 7809-7814.

Widholm, J. M. (1972). Tryptophan biosynthesis in Nicotiana tabacum and Daucus carota cell cultures: Site of action of inhibitory tryptophan analogues. Biochimica et Biophysica Acta, $261,44-51$.

Woodward, A. W., \& Bartel, B. (2005). Auxin: Regulation, action and interaction. Annals of Botany, 95, 707-735.

Yamada, T., Tozawa, Y., Hasegawa, H., Terakawa, T., Ohkawa, Y., $\&$ Wakasa, K. (2004). Use of a feedback-insensitive $\alpha$ subunit of anthranilate synthase as a selectable marker for transformation of rice and potato. Molecular Breeding, 14, 363-373.

Yao, K., De Luca, V., \& Brisson, N. (1995). Creation of a metabolic sink for tryptophan alters the phenylpropanoid pathway and the susceptibility of potato to phytophthora infestans. Plant Cell, 7 , $1787-1799$.

Zhang, X. -H., Brotherton, J. E., Song, H. -S., Widholm, J. M., Portis, A. R., Jr. (2001). Targeting a nuclear anthranilate synthase alpha-subunit gene to the tobacco plastid genme results in enhanced tryptophan biosynthesis. Return of gene to its preendosymbiotic origin. Plant Physiology, 127, 131-141.

Zhao, T., Christensen, S. K., Frankhauser, C., Cashman, J. R., Cohen, J. D., Weigel, D., \& Chory, J. (2001). A role for flavin monooxygenase-like enzymes in auxin biosynthesis. Science, 291, 306-309.

Zhao, Y., Hull, A. K., Gupta, N. R., Goss, K. A., Alonso, J., Ecker, J. R., Normanly, J., Chory, J., \& Celenza, J. L. (2002). Trpdependent auxin biosynthesis in Arabidopsis: Involvement of cytochrome P450s CYP79B2 and CYP79B3. Gene Development, 16, 3100-3112. 